\title{
NONLINEAR MODEL PREDICTIVE CONTROL OF BATCH PROCESSES: AN INDUSTRIAL CASE STUDY
}

\author{
Zoltan K. Nagy ${ }^{\dagger 1}$, Bernd Mahn ${ }^{\times}$, Rüdiger Franke ${ }^{\ddagger}$, Frank Allgöwer ${ }^{\dagger}$, \\ ${ }^{\dagger}$ University of Stuttgart, Stuttgart, Germany \\ ${ }^{\ddagger} A B B$ Corporate Research, Ladenburg, Germany \\ ${ }^{\times}$BASF Aktiengesellschaft, Ludwigshafen, Germany
}

\begin{abstract}
Batch processes play a significant role in the production of most modern highvalue added products. The paper illustrates the benefits of nonlinear model predictive control (NMPC) for the setpoint tracking control of an industrial batch polymerization reactor. Real-time feasibility of the on-line optimization problem from the NMPC is achieved using an efficient multiple shooting algorithm. A real-time formulation of the NMPC that takes computational delay into account is described. The control relevant model used in the NMPC is derived from the complex first principles model and is fitted to the experimental data using maximum likelihood estimation. A parameter adaptive extended Kalman filter (PAEKF) is used for state estimation and on-line model adaptation. The performance of the NMPC implementation is assessed via simulation and experimental results. Copyright $\odot 2005$ IFAC
\end{abstract}

Keywords: Industrial control, maximum likelihood estimation, multiple shooting algorithm, nonlinear model predictive control, parameter adaptive extended Kalman filter, parameter estimation, state estimation, real-time control.

\section{INTRODUCTION}

The inherent advantages of batch processes, including their ability to produce multiple related products in the same facility, as well as their ability to handle variations in feed stocks, product specifications and market demand patterns, makes them well suited for the manufacture of low-volume, high-value products. For these reasons, batch processes are the production scheme of choice for the pharmaceutical, biotechnology, specialty chemical, consumer products, agricultural chemical, and microelectronics industries. The production of these high value-added chemicals, today contributes a

\footnotetext{
${ }^{1}$ Curresponding author. University of Stuttgart, Pfaffenwaldring 9, 70550, Stuttgart, Germany, Phone: +49-711-6857742; Fax:+49-711-6857735; Email: nagy@ist.uni-stuttgart.de
}

significant and growing portion of the revenue and earnings of the chemical process industries. Due to the highly competitive and profit driven nature of today's process industries, nonlinear model predictive control (NMPC) techniques are becoming increasingly accepted, being one of the approaches that inherently can cope with process constraints, nonlinearities, and different objectives derived from economical or environmental considerations (Bequette, 1991; Morari and Lee, 1997; Henson, 1998). However, despite the significant and continuously increasing importance of batch processes, the number of NMPC applications is significantly lower than in the case of continuous processes (Qin and Badgwell, 2003). Although the inherent nonlinearity of batch processes suggests NMPC as a natural choice for the advanced control of these systems, most industrial NMPC vendors for example do not support typical batch NMPC problems. This can be explained mainly by the special features of batch processes that make their 
control very challenging. Generally, in batch process operation, two different control problems arise:

(a) End-point property control (setpoint optimization approach). In the case of batch processes the real economic objective is usually related to the product quality at the end of the batch, leading to the formulation of a control problem in terms of economic or performance objective at the end of the batch, which is always implemented in a shrinking horizon approach.

(b) Setpoint tracking, when off-line or on-line determined, usually time-varying setpoint trajectories have to be followed in a moving or shrinking (or combination of the two) horizon approach. In this case usually a quadratic (least squares type) objective function is used.

Most of the applications in advanced batch process control deal with the tight temperature regulation. Although this is a typical control problem, it presents a major difference compared to the operation of continuous processes, since important properties such as Lyapunov stability are no longer defined. Controller performance can only be assessed based on the chosen objective function, existing constraints and robustness against model/plant mismatch or control implementation uncertainties. Additionally, in the case of continuous processes computational burden can be reduced by choosing a shorter control horizon then prediction horizon, whereas in the case of batch NMPC the control and prediction horizons should be chosen equal, to avoid large deviations of the predicted quantities from their (usually timevarying) setpoints due to the transient character of the process, increasing significantly the computational demand. In this paper an efficient real-time NMPC is applied to an industrial batch polymerization reactor. The approach exploits the advantages of an efficient optimization algorithm based on multiple shooting technique (Franke and Arnold; Diehl, 2001) to achieve real-time feasibility of the on-line optimization problem involved in the NMPC, even in the case of the large control and prediction horizon. The complex first principle model of the process is used off-line to determine the optimal temperature profile that provides the required quality and conversion in minimum time. The NMPC is used for tight setpoint tracking of the optimal temperature profile. Based on the available measurements the complex model is not observable hence cannot be used directly in the NMPC strategy. To overcome the problem of unobservable states, a grey-box modelling approach is used, where some unobservable parts of the model are described through nonlinear empirical relations, developed from the detailed first-principles model. The resulted control-relevant model is fine tuned using experimental data and maximum likelihood estimation. A parameter adaptive extended Kalman filter (PAEKF) is used for state estimation and online parameter adaptation to account for mode/plant mismatch.

\section{COMPUTATIONALY EFFICIENT REAL-TIME OUTPUT FEEDBACK NMPC}

\subsection{Problem formulation}

Nonlinear model predictive control is an optimization-based multivariable constrained control technique that uses a nonlinear dynamic model for the prediction of the process outputs. At each sampling time the model is updated on the basis of new measurements and state variable estimates. Then the open-loop optimal manipulated variable moves are calculated over a finite prediction horizon with respect to some cost function, and the manipulated variables for the subsequent prediction horizon are implemented. Then the prediction horizon is shifted or shrunk by usually one sampling time into the future, and the previous steps are repeated.

The optimal control problem to be solved on-line in every sampling time in the NMPC algorithm can be formulated as:

Problem $P 1$ :

$$
\min _{u(t) \in \mathcal{U}} \mathcal{H}(x(t), u(t) ; \theta)
$$

subject to:

$$
\begin{aligned}
& \dot{x}(t)=f(x(t), u(t) ; \theta), \\
& y(t)=g(x(t), u(t) ; \theta), \\
& x\left(t_{k}\right)=\hat{x}\left(t_{k}\right), \quad x\left(t_{0}\right)=\hat{x}_{0}, \\
& h(x(t), u(t) ; \theta) \leq 0, \quad t \in\left[t_{k}, t_{F}\right],
\end{aligned}
$$

where $\mathcal{H}$ is the performance objective, $t$ is the time, $t_{k}$ is the time at sampling instance $k, t_{F}$ is the final time at the end of prediction, $x(t) \in \mathbb{R}^{n_{x}}$ is the $n_{x}$ vector of states, $u(t) \in \mathcal{U}$ is the $n_{u}$ set of input vectors, $y(t) \in \mathbb{R}^{n_{y}}$ is the $n_{y}$ vector of measured variables used to compute the estimated states $\hat{x}\left(t_{k}\right)$, $\theta \in \Theta \subset \mathbb{R}^{n_{\theta}}$ is the $n_{\theta}$ vector of possible uncertain parameters, where the set $\Theta$ can be either defined by hard bounds or probabilistic, characterized by a multivariate probability density function. The function $f: \mathbb{R}^{n_{x}} \times \mathcal{U} \times \Theta \rightarrow \mathbb{R}^{n_{x}}$ is the twice continuously differentiable vector function of the dynamic equations of the system, $g: \mathbb{R}^{n_{x}} \times \mathcal{U} \times \Theta \rightarrow \mathbb{R}^{n_{y}}$ is the measurement equations function, and $h: \mathbb{R}^{n_{x}} \times \mathcal{U} \times \Theta \rightarrow \mathbb{R}^{c}$ is the vector of functions that describe all linear and nonlinear, timevarying or end-time algebraic constraints for the system, where $c$ denotes the number of these constraints. The objective function can have the following general form:

$$
\mathcal{H}(x(t), u(t) ; \theta)=\mathcal{M}\left(x\left(t_{F}\right) ; \theta\right)+\int_{t_{k}}^{t_{F}} \mathcal{L}(x(t), u(t) ; \theta) d t
$$

We assume that $\mathcal{H}: \mathbb{R}^{n_{x}} \times \mathcal{U} \times \Theta \rightarrow \mathbb{R}$ is twice continuously differentiable, thus fast optimization algorithms, based on first and second order derivatives may be exploited in the solution of (6). The form of (6) is general enough to express a wide range of objectives encountered in NMPC applications (moving or shrinking horizon approach on regulation and/or setpoint tracking, direct 
minimization of the operation time, optimal initial conditions, multiple simultaneous objectives, treatment of soft constraints, terminal penalty term for stability, etc.). For batch processes with end-point optimization the objective usually reduces to the Mayer form $(\mathcal{L}(\cdot)=0)$, however the Lagrange term $(\mathcal{L}(\cdot))$ still may be used, e.g. to implement soft constraints on control rate. In NMPC the optimization problem (1)-(5) is solved iteratively online, in a moving (receding) horizon $\left(t_{F}<t_{f}\right)$ or shrinking horizon $\left(t_{F}=t_{f}\right)$ approach, where $t_{f}$ is the batch time.

\subsection{Efficient solution of the NMPC optimization via multiple shooting}

Considering the discrete nature of the on-line control problem, the continuous time optimization problem involved in the NMPC formulation is solved by formulating a discrete approximation to it, that can be handled by conventional nonlinear programming (NLP) solvers (Biegler, 2000). The time horizon $t \in\left[t_{0}, t_{f}\right]$ is divided into $N$ equally spaced time intervals $\Delta t$ (stages), with discrete time steps $t_{k}=t_{0}$ $+k \Delta t$, and $k=0,1, \ldots, N$. Model equations are discretized,

$$
x_{k+1}=f_{k}\left(x_{k}, u_{k} ; \theta\right)
$$

and added to the optimization problem as equality constraints. Problem $P 2$ gives the general discrete time formulation of Problem $P 1$.

Problem P2:

$$
\min _{u_{k}, u_{k+1}, ., u_{k+N_{p}}}\left\{\mathcal{M}^{k+N_{p}}\left(x_{k+N_{p}} ; \theta\right)+\sum_{j=k}^{k+N_{p}} \mathcal{L}_{j}\left(x_{j}, u_{j} ; \theta\right)\right\},
$$

subject to:

$$
\begin{gathered}
G_{k}\left(x_{k}, u_{k} ; \theta\right)=0, \\
H_{k}\left(x_{k}, u_{k} ; \theta\right) \leq 0,
\end{gathered}
$$

where $N_{p}$ is the number of stages in the prediction horizon $\left[t_{k}, t_{F}\right], G_{k}: \mathbb{R}^{n_{x}} \times \mathcal{U} \times \Theta \rightarrow \mathbb{R}^{n_{x}+n_{y}}$ corresponds to all equality constraints resulted from (7) and (3), $H_{k}: \mathbb{R}^{n_{x}} \times \mathcal{U} \times \Theta \rightarrow \mathbb{R}^{c+2 n_{u}}$ is the vector function of all inequality constraints (5), including the constraints on the inputs considering. We consider here that the set of possible inputs is given as hard bounds. It is assumed that the vector functions $G$ and $H$ are twice continuously differentiable.

A very efficient solution technique for the problem (8)-(10) is based on the multiple shooting approach (Diehl, 2001; Frake and Arnold). This procedure consists of dividing up the time interval $t \in\left[t_{0}, t_{f}\right]$ into a series of grid points $\left[t_{0}, t_{1}, t_{2}, \ldots, t_{f}\right]$. Note that the grid points do not necessary correspond to the discretization points in the definition of problem $P 2$. Using a local control parameterizations a shooting method is performed between successive grid points (see Figure 2). The differential equations and cost on these intervals are integrated independently during each optimization iteration, based on the current guess of the control. The continuity/consistency of the final state trajectory at the end of the optimization is enforced by adding consistency constraints to the nonlinear programming problem. A set of starting values for the state and adjoint vectors is required at each grid point in time, and continuity conditions for the solution trajectory introduce additional interior boundary conditions, which are incorporated into one large zero-finding problem to be solved. The solution of Problem $P 2$ is performed using an NMPC tool (Nagy et al., 2004) based on the sequential-quadratic-programming (SQP) type optimizer HQP, which is used in conjunction with the implicit differential-algebraic-equation (DAE) solver, DASPK, for robust and fast solution of the model equations.

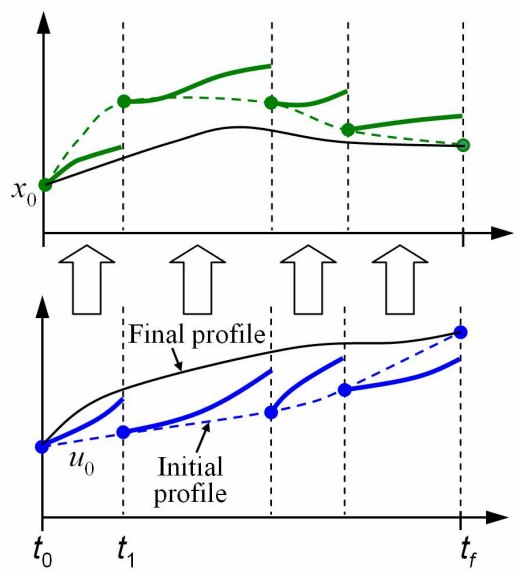

Fig. 1. Illustration of the multiple shooting approach.

\subsection{Real-time NMPC algorithm}

The solution of problem $P 2$ requires a certain, usually not negligible, amount of computation time $\delta_{k}$, while the system will evolve to a different state. In this case the optimal feedback control $u^{\star}\left(t_{k}\right)=\left[u_{0 \mid t_{k}}, u_{1 \mid t_{k}}, \ldots, u_{N_{p} \mid t_{k}}\right]$ computed in moment $t_{k}$ corresponding to the information available up to this moment, will no longer be optimal. Computational delay $\delta_{k}$ has to be taken into consideration in realtime applications. In the approach used here, in moment $t_{k}$, first the control input from the second stage of the previous optimization problem $u_{1 \mid t_{t-1}}$ (which corresponds to the first stage of the current optimization) is injected into the process, and then the solution of the current optimization problem is started, with fixed $u_{0 \mid t_{k}}=u_{1 \mid t_{k-1}}$. After completion, the optimization idles for the remaining period of $t \in\left(t_{k}+\delta_{k}, t_{k+1}\right)$, and then at the beginning of the next stage, at moment $t_{k+1}=t_{k}+\Delta t, \quad u_{1 \mid t_{k}}$ is introduced into the process, and the algorithm is repeated. This approach requires real-time feasibility for the solution of each open-loop optimization problems $\left(\delta_{k} \leq \Delta t\right)$.

\subsection{State estimation}

Proper state estimation is crucial for the success of the NMPC application. Extended Kalman filter (EKF) has been widely used in process control applications, however its performance strongly depends on the accuracy of the model. To avoid highly biased model predictions, some of the model parameters are estimated together with the states, 
leading to a parameter adaptive EKF formulation (PAEKF) (Valappil and Georgakis, 2002). Define $\theta^{\prime} \subseteq \theta$ as the subset of the estimated parameters from the parameter vector, and $\theta^{\prime \prime} \triangleq \theta \backslash \theta^{\prime}$ the set of the remaining parameters. The augmented state vector in this case is given by $\mathcal{X}=\left[x, \theta^{\prime}\right]^{T}$, and the augmented model used for estimation is given by

$$
\dot{\mathcal{X}}=\left[f\left(x, \theta^{\prime}, u ; \theta^{\prime \prime}\right), \mathbf{0}\right]^{T}+\left[w, w_{\theta^{\prime}}\right]^{T},
$$

where $w$, and $w_{\theta^{\prime}}$ are zero-mean Gaussian white noise variables. The time-varying state space matrix of the locally linearized augmented model is defined as follows:

$$
\mathcal{A}\left(t_{k}\right)=\left[\begin{array}{cc}
\frac{\partial f\left(x\left(t_{k}\right), \theta^{\prime}\left(t_{k}\right), u\left(t_{k}\right) ; \theta^{\prime \prime}\right)}{\partial x} & \frac{\partial f\left(x\left(t_{k}\right), \theta^{\prime}\left(t_{k}\right), u\left(t_{k}\right) ; \theta^{\prime \prime}\right)}{\partial \theta^{\prime}} \\
\mathbf{0} & \mathbf{0}
\end{array}\right]
$$

The measurement covariance matrix is determined based on the accuracy of the measurements. The appropriate choice of the state covariance matrix, $\mathbf{Q}$, is however often difficult in practical applications. An estimate of $\mathbf{Q}$ can be obtained by assuming that the process noise vector mostly represents the effects of parametric uncertainty (Valapil and Georgakis, 2000). Based on this assumption and performing a first-order power series expansion of the model error equations using the nominal parameter vector and control trajectory, the process noise covariance matrix can be computed as follows:

$$
\mathbf{Q}(t)=\mathbf{S}_{\theta}(t) \mathbf{V}_{\theta} \mathbf{S}_{\theta}^{T}(t)
$$

where $\mathbf{V}_{\theta} \in \mathbb{R}^{n_{\theta} \times n_{\theta}}$ is the parameter covariance matrix, and $\mathbf{S}_{\theta}(t)$ is the jacobian computed using the nominal parameters and estimated states:

$$
\mathbf{S}_{\theta}(t)=\left(\frac{\partial f}{\partial \theta}\right)_{\hat{x}(t), u(t), \hat{\theta}}
$$

Equation (13) provides an easily implementable way to estimate the process noise covariance matrix, since the parameter covariance matrix $\mathbf{V}_{\theta}$ is usually available from parameter estimation, and the sensitivity coefficients in $\mathbf{S}_{\theta}(t)$ can be computed by finite differences or via sensitivity equations (Feehery et al, 1997). Note that the above approach leads to a time-varying, full covariance matrix, which has been shown to provide better estimation performance for batch processes than the classically used constant, diagonal Q ( Valapil and Georgakis, 2000; Nagy and Braatz, 2003).

\section{SETPOINT TRACKING NMPC OF THE INDUSTRIAL BATCH REACTOR}

A schematic representation of the experimental pilot plant is shown on Figure 2. The reactor temperature is controlled using a complex heating-cooling system, which is based on a closed oil circuit, which is recycled through the jacket with a constant flow rate $F_{j}$. The heating-cooling medium goes through a multi-tubular heat exchanger where a PI controller tries to keep the temperature difference constant by adjusting the cooling water flow rate. Heating is performed using an electric heater. The power of the heater is adjusted by the lower level PI controller that controls the input temperature into the jacket. The setpoint of the PI controller is determined by the higher level NMPC that has the objective to track a predetermined temperature profile in the reactor.

\subsection{Modelling and identification of the reactor}

A detailed first-principles model of the process containing material and energy balances as well as detailed kinetic and thermodynamic models was developed and identified based on off-line experiments. Since only temperature measurements are available in the plant, many states of the detailed model are not estimable, or not even detectable. The complex model however was used to determine the optimal temperature profile, and for deriving the control-relevant model. Available measurements are: reactor temperature $\left(T_{r}\right)$, and input and output temperatures into and from the jacket, $\left(T_{j i n}, T_{j}\right)$. With this set of measurements the following reduced model was used in the NMPC:

$$
\begin{gathered}
\dot{n}_{M}=-Q_{r} / \Delta H_{r} \\
\dot{T}_{r, k}=\left(Q_{r}+U_{w} A_{w}\left(T_{w, k}-T_{r, k}\right)-(U A)_{\text {loss }, r}\left(T_{r, k}-T_{a m b}\right)\right) \\
\quad /\left(m_{M} c_{p, M}+m_{P} c_{p, P}+m_{\text {uater }} c_{p, u a t e r}\right) \\
\dot{T}_{w, k}=\left(U_{j} A_{j}\left(T_{j, k}-T_{w, k}\right)-U_{w} A_{w}\left(T_{w, k}-T_{r, k}\right)\right) / m_{w} / c_{p w} \\
\dot{T}_{j, k}=\left(\mathcal{N} F_{j} \rho_{j} c_{p, j}\left(T_{j, k-1}-T_{j, k}\right)-U_{j} A_{j}\left(T_{j, k}-T_{w, k}\right)\right. \\
\left.\quad-(U A)_{l o s s, j}\left(T_{j, k}-T_{a m b}\right)\right) /\left(m_{j} c_{p, j}\right)
\end{gathered}
$$

where $k=1, \ldots, \mathcal{N}, T_{r}=T_{r, \mathcal{N}}, T_{j}=T_{j, \mathcal{N}}, T_{j, 0}=T_{j i n}$, $n_{M}$ is the number of mol of monomer, $\Delta H_{r}$ is the enthalpy of reaction, $T_{w}$ is the wall temperature, $U$ and $A$ are heat transfer coefficients and areas from reactor to wall $(\cdot)_{w}$ or wall to jacket $(\cdot)_{j}$, $c_{p, M / P / \text { water } / w / j}$ and $m_{M / P / \text { water } / w / j}$ are the heat capacities and masses of monomer, polymer, water, wall and oil, $T_{a m b}$ is the ambient temperature, $\rho_{j}$ is the density of the oil, $(U A)_{\text {loss }, r / j}$ heat loss coefficients in the reactor and jacket, respectively.

To estimate the transport delay, the reactor, wall and jacket were divided in $\mathcal{N}=4$ elements, leading to a system of 13 differential equations. For proper temperature control the estimation of the generated

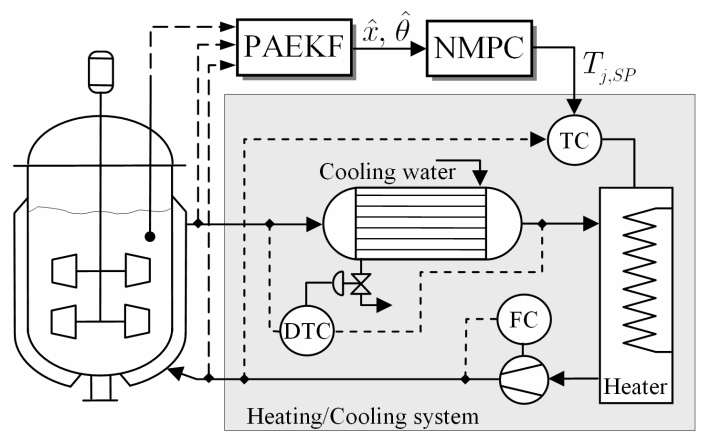

Fig. 2. Schematic representation of the batch reactor with the heating/cooling system. 


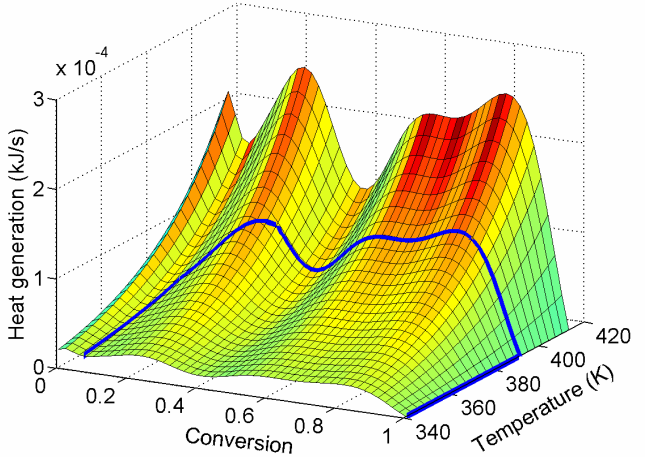

Fig. 3. Heat generation surface obtained from the detailed model. The solid line represents the heat generation corresponding to the desired temperature trajectory.

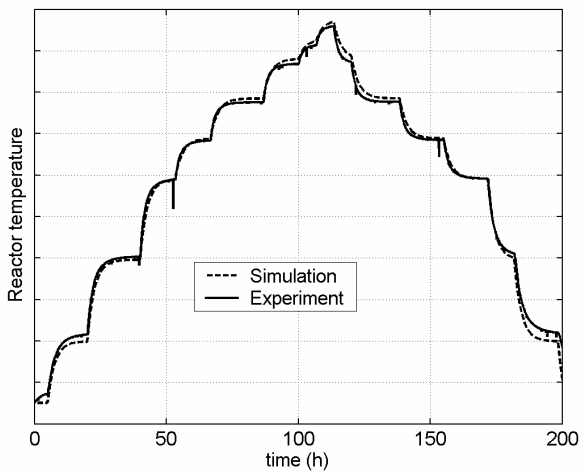

Fig. 4. Validation of the model compared to plant data.

heat $Q_{r}$ is important, therefore an empirical nonlinear relation $Q_{r}=f_{Q}\left(n_{M}, T_{r}\right)$ was determined from the complex first principle model, simulating the process for different temperature profiles. Figure 3 shows the heat generation surface obtained from the empirical model and the heat generated when the desired temperature trajectory is followed.

Maximum likelihood estimation was used to fit the parameters of the model (15)-(18) to the data obtained from the plant, performing several water batches (when $Q_{r}=0$ ), and minimizing the objective function below:

$$
\psi_{M L E}(\theta)=\left(n_{\exp } / 2\right) \sum_{i=1}^{n_{\text {meas }}} \ln \left(\sum_{j=1}^{n_{\text {exp }}} \varepsilon_{i j}(\theta)\right)
$$

where $n_{\exp }$ is the number of experimental points, from each of the $n_{\text {meas }}$ sensors, and $\varepsilon_{i j}$ is the error between the measurement and model prediction. The following parameter vector was used in the estimation:

$$
\theta=\left[(U A)_{l o s s, r},(U A)_{l o s s, j}, U_{j} A_{j}, m_{w}, U_{w} A_{w}, m_{j}\right]
$$

This procedure gives the optimal nominal parameter estimates, $\hat{\theta}^{\star}$, and the corresponding uncertainty description given by the covariance matrix, estimated from the Hessian of the objective (19) at the optimal parameter estimate, $\mathbf{V}_{\theta} \approx H^{\star^{-1}}=\left(\partial^{2} \psi / \partial \theta^{2}\right)_{\theta=\hat{\theta}^{\star}}^{-1}$. The good fit between the experimental data end the model is shown on Figure 4.

\subsection{NMPC of the bath reactor}

Model (15)-(18) was used in an adaptive output feedback NMPC approach, where the objective was to provide a tight setpoint tracking, by minimizing online, in every sampling instance $k$, the following quadratic objective:

$$
\min _{u(t)} \int_{t_{k}}^{t_{F}}\left(\left(T_{r}(t)-T_{r}^{r e f}(t)\right)^{2}+Q_{\Delta u} d u(t)^{2}\right) d t
$$

The optimal setpoint profile $T_{r}^{r e f}$ was obtained via off-line optimization using the detailed model. The manipulated input of the NMPC , $u(t)=T_{j S P}$, is the setpoint temperature to the lower level PI controller, which controls the input temperature into the jacket. Real-time simulations were performed when the complex model was used to simulate the real plant on one computer, whereas the NMPC was running on a different computer. The communication between the plant and NMPC was performed via the standard OPC interface, simulating the real industrial setup. The performance of the NMPC is presented on Figures 5 and 6. The grey bands in the figures represent the set of model predictions and control inputs during the batch. Figure 5 demonstrates the very good setpoint tracking performance of the controller and prediction abilities of the model, which has been adapted during the batch. The parameters $\theta^{\prime}=\left[Q_{r}, U_{w} A_{w}\right]$ were estimated together with the model states in the PAEKF. The parameter covariance matrix $\mathbf{V}_{\theta}$, resulted from the identification was used to compute the state covariance matrix in the estimator according to (13). This approach provides a good estimate of the generated heat, shown in Figure 7. A weighting coefficient of $Q_{\Delta u}=0.4$, and prediction and control horizons of $8000 s$ were used, in the optimization, with a sampling time of $20 \mathrm{~s}$. The control input was discretized in 400 piecewise constant inputs, leading to a significantly high dimensional optimization problem. The efficient multiple shooting approach guarantees the real-time feasibility of the NMPC implementation. Even with the large control discretization of 400 the computation time was below the sampling time of $20 s$, as can be seen in Figure 8 . The larger control error during the first half of the constant temperature profile (or the end of the batch) is caused by the saturation of the lower level PI controller, which can also be observed on Figure 6 . In these periods of the batch the setpoint values given by the NMPC can not be achieved. This error can be eliminated only if the lower level PI controller is introduced in the prediction model.

Similar conclusions have been achieved from the experiments conducted in the real plant located at BASF in Ludwigshafen, Germany. Initial results predict the good performance of the NMPC shown on Figure 9. The experiments demonstrate the improvement in the control performance if the lower level controller is modelled (in scenarios when saturation can occur) and illustrate the necessity of proper online model adaptation, when the complex cooling-heating system is not modelled in detail. 


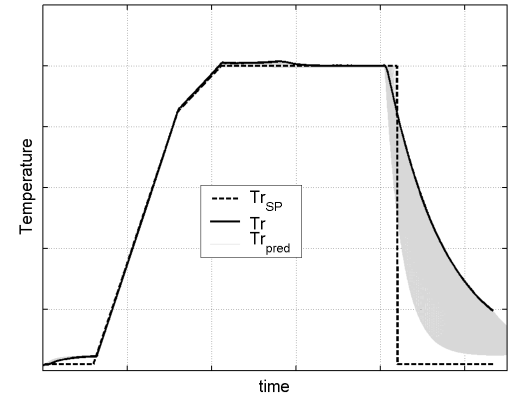

Fig. 5. Setpoint tracking performance of the NMPC, and model predictions during the batch.

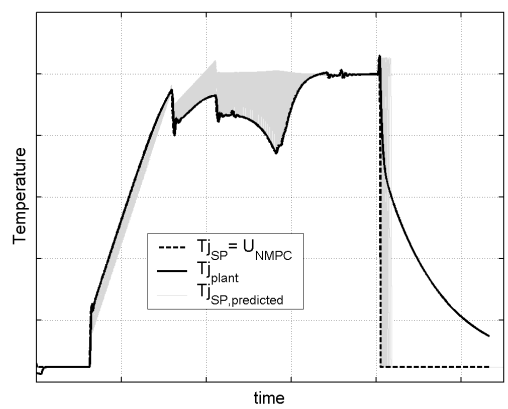

Fig. 6. NMPC control input profiles ( $\left.T_{j . S P \text {.predicted }}\right)$, the finally implemented control input $\left(T_{j, S P}\right)$, and the real jacket temperature $T_{j, p l a n t}$.

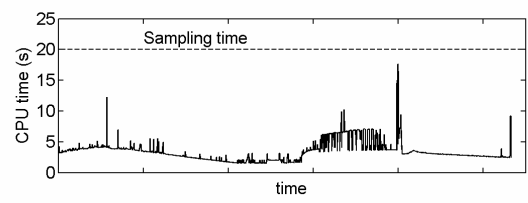

Fig. 7. Total CPU times (estimation + optimization) along the batch.

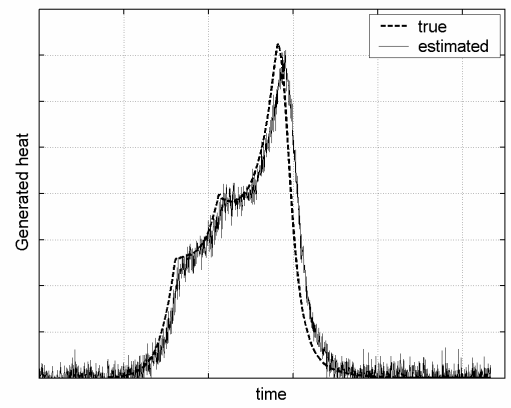

Fig. 8. Estimated and real reaction heat $\left(Q_{r}\right)$.
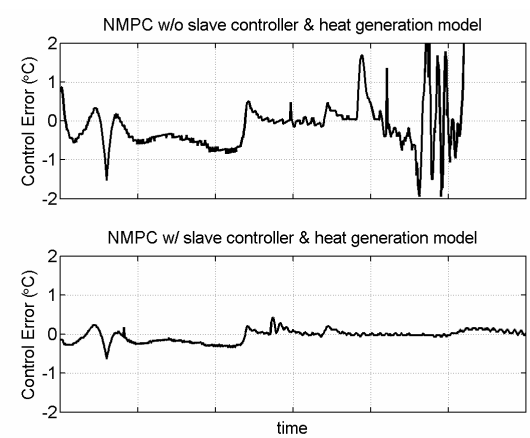

Fig. 9. Tracking error of the NMPC in the case of two real plant experiments: without (upper plot) and with (lower plot) the prediction of heat generation and slave controller model.

\section{CONCLUSIONS}

The paper present a computationally efficient NMPC approach that combines output feedback design with efficient optimization technique to provide a nonlinear model predictive control approach that can be supported in an industrial environment. Detailed first-principles model is used to derive the reduced control-relevant model based on the available measurements, which is tuned using data from the plant, and used then in the NMPC. A PAEKF is combined with the control algorithm for the on-line state estimation and model adaptation to achieve offset free control. Simulation and experimental results demonstrate the efficiency of the NMPC approach in an industrial application. The approach presented here is applicable to a generic class of batch systems with exothermic reaction, requiring only the determination of the proper heat generation function in each case (and model parameters if other reactor is used), which can be obtained either from a detailed first-principles model or from plant data in an iterative learning framework.

\section{REFERENCES}

Biegler, L., (2000). Efficient solution of dynamic optimization and NMPC problems. In F. Allgöwer and A. Zheng (Ed), Nonlinear Predictive Control, Bagel, Birkhauser.

Bequette, B.W. (1991). Nonlinear Control of Chemical Processes - A Review. Ind. Eng. Chem. Res., 30, 1391-1413.

M. Diehl (2001). Real-Time Optimization for Large Scale Nonlinear Processes. PhD Thesis, University of Heidelberg.

Feehery, W. F., J. E. Tolsma and P. I. Barton (1997). Efficient sensitivity analysis of large-scale differential-algebraic systems. Appl. Numer. Math., 25, 41-54.

Franke, R., E. Arnold and H. Linke. HQP: A solver for nonlinearly constrained large-scale optimization. http://hqp.sourceforge.net.

Henson, A.M. (1998). Nonlinear model predictive control: current status and future directions. Comp. Chem. Eng., 23, 187-201.

Nagy, Z.K. and R.D. Braatz (2003). Robust nonlinear model predictive control of batch processes. AIChE J., 49, 1776-1786.

Nagy, Z.K., F. Allgower, R. Franke, A. Frick and B. Mahn (2004). Efficient tool for nonlinear model predictive control of batch processes. in Proc. of the 12th Mediterranean Conf. on Cont. and Aut. (MED'04), Kusadasi, Turkey.

Morari, M. and J.H. Lee (1997). Model predictive control: Past, present and future. In Proc. PSE'97-ESCAPE-7 Symposium, Trondheim.

Qin S. J., and T. Badgewell (2003). A Survey of Industrial Model Predictive Control Technology. Control Engineering Practice, 11, 733-764.

Valappil, J. and C. Georgakis (2000). Systematic estimation of state noise statistics for Extended Kalman Filters. AIChE J., 46, 292-308. 